\title{
Article \\ Enhanced Properties of Extended Wavelength InGaAs on Compositionally Undulating Step-Graded InAsP Buffers Grown by Molecular Beam Epitaxy
}

\author{
Xuefei Li ${ }^{1,2, \dagger}$, Jianming $X u^{3,+}$, Tieshi Wei ${ }^{1,2}$, Wenxian Yang ${ }^{2}$, Shan Jin ${ }^{2}$, Yuanyuan Wu ${ }^{2}$ and Shulong Lu ${ }^{2, *}$ \\ 1 School of Nano-Tech and Nano-Bionics, University of Science and Technology of China, Hefei 230026, China; \\ xfli2011@sinano.ac.cn (X.L.); tswei2020@sinano.ac.cn (T.W.) \\ 2 Key Lab of Nanodevices and Applications, Suzhou Institute of Nano-Tech and Nano-Bionics, \\ Chinese Academy of Sciences (CAS), Suzhou 215123, China; wxyang2014@sinano.ac.cn (W.Y.); \\ sjin2017@sinano.ac.cn (S.J.); yywu2011@sinano.ac.cn (Y.W.) \\ 3 State Key Laboratory of Space Power-Sources Technology, Shanghai Institute of Space Power-Sources, \\ Shanghai 200245, China; xujianming1127@163.com \\ * Correspondence: sllu2008@sinano.ac.cn \\ + These authors contributed equally to this work.
}

Citation: Li, X.; Xu, J.; Wei, T.; Yang, W.; Jin, S.; Wu, Y.; Lu, S. Enhanced Properties of Extended Wavelength InGaAs on Compositionally Undulating Step-Graded InAsP Buffers Grown by Molecular Beam Epitaxy. Crystals 2021, 11, 1590. https://doi.org/10.3390/ cryst 11121590

Academic Editors: Daohua Zhang, Dawei Zhang, Jinchao Tong and Fei Suo

Received: 29 November 2021 Accepted: 17 December 2021 Published: 20 December 2021

Publisher's Note: MDPI stays neutral with regard to jurisdictional claims in published maps and institutional affiliations.

Copyright: (c) 2021 by the authors. Licensee MDPI, Basel, Switzerland. This article is an open access article distributed under the terms and conditions of the Creative Commons Attribution (CC BY) license (https:// creativecommons.org/licenses/by/ $4.0 /)$.
Abstract: The extended wavelength InGaAs material $(2.3 \mu \mathrm{m})$ was prepared by introducing compositionally undulating step-graded $\operatorname{InAs} \mathrm{P}_{1-y}$ buffers with unequal layer thickness grown by solid-source molecular beam epitaxy (MBE). The properties of the extended wavelength InGaAs layer were investigated. The surface showed ordered crosshatch morphology and a low roughness of $1.38 \mathrm{~nm}$. Full relaxation, steep interface and less than one threading dislocation in the InGaAs layer were demonstrated by taking advantage of the strain compensation mechanism. Room temperature photoluminescence (PL) exhibited remarkable intensity attributed to the lower density of deep non-radiative centers. The emission peak energy with varied temperatures was in good agreement with Varshni's empirical equation, implying high crystal quality without inhomogeneity-induced localized states. Therefore, our work shows that compositionally undulating step-graded InAsP buffers with a thinner bottom modulation layer, grown by molecular beam epitaxy, is an effective approach to prepare InGaAs materials with wavelengths longer than $2.0 \mu \mathrm{m}$ and to break the lattice limitation on the materials with even larger mismatch.

Keywords: extended wavelength InGaAs; photoluminescence; strain compensation; InAsP buffer

\section{Introduction}

The ternary $\operatorname{In}_{x} \mathrm{Ga}_{1-\mathrm{x}}$ As alloy has gained great success on optoelectronic applications due to the tunable bandgap from $0.9 \sim 3 \mu \mathrm{m}$, which is corresponding to the atmosphere transmission window [1,2]. For example, $\mathrm{In}_{0.53} \mathrm{Ga}_{0.47} \mathrm{As}$, which is lattice-matched to $\operatorname{InP}$ materials, has been widely used in multi-junction photovoltaics and commercially available in opto-communications [3-6]. In recent years, extended wavelength $\operatorname{In}_{x} \mathrm{Ga}_{1-x} \mathrm{As}(\mathrm{x}>0.53)$ and derived quaternary alloy have attracted more and more attention in thermophotovoltaics (TPV), remote sensing, and thermal imaging [7-12]. The lattice mismatch to InP substrate can be up to $3.2 \%(x=1)$, and misfit dislocation will be generated to relax strains in the active epilayer during growth, which will deteriorate the device performances [13]. At this point, appropriate buffers with moderate thickness, enough relaxation and low density of dislocation must be applied to migrate the lattice mismatch. Many efforts have been taken to improve the quality of the $\operatorname{In}_{x} \mathrm{Ga}_{1-x} \mathrm{As}$ epilayer. Some researchers used linear graded buffers to stop the propagation of dislocation and others took strained superlattice to engineer the strain field [14-18]. Compositionally step-graded buffers were also employed. $\mathrm{In}_{\mathrm{x}} \mathrm{Al}_{1-\mathrm{x}} \mathrm{As}$ and $\mathrm{InAs}_{\mathrm{y}} \mathrm{P}_{1-\mathrm{y}}$ were commonly used materials. $\mathrm{Gu}$ et al. studied properties of $\mathrm{In}_{0.85} \mathrm{Ga}_{0.15} \mathrm{As}$ on different $\mathrm{In}_{\mathrm{x}} \mathrm{Al}_{1-\mathrm{x}} \mathrm{As}$ buffer structures systematically grown by gas-source 
molecular beam epitaxy (GSMBE) at several advantages, and $\operatorname{In}_{0.85} \mathrm{Ga}_{0.15}$ As photodetectors with cutoff at $2.5 \mu \mathrm{m}$ were made based on device-quality epilayer growth $[14,19]$. Hudait et al. reported $\operatorname{In}_{0.69} \mathrm{Ga}_{0.31} \mathrm{As}$ TPV device with step-graded $\operatorname{In} A \mathrm{~s}_{\mathrm{y}} \mathrm{P}_{1-\mathrm{y}}$ buffer, and compared the $\operatorname{In}_{0.69} \mathrm{Ga}_{0.31}$ As properties with $\operatorname{In}_{x} \mathrm{Al}_{1-\mathrm{x}} \mathrm{As}$ and $\mathrm{InAs}_{\mathrm{y}} \mathrm{P}_{1-\mathrm{y}}$ buffers grown by solid-source molecular beam epitaxy (SSMBE), showing that InAs $\mathrm{P}_{1-\mathrm{y}}$ displayed ideal characteristics, while $\operatorname{In}_{x} \mathrm{Al}_{1-x}$ As presented phase decomposition and rougher surface [20]. Our group have reported $0.6 \mathrm{eV} \operatorname{In}_{0.69} \mathrm{Ga}_{0.31} \mathrm{As}$ TPV device (corresponding band-edge emission at $2.0 \mu \mathrm{m}$ ) on compositionally undulating step-graded $\operatorname{In} \mathrm{As}_{\mathrm{y}} \mathrm{P}_{1-\mathrm{y}}$ buffer with equal layer thickness grown by metal organic chemical vapor deposition (MOCVD), and a lower surface roughness of $2.74 \mathrm{~nm}$ was achieved than that on conventional step-graded buffers [8,9]. For further extend wavelength, thicker buffers would be taken and more indium must be incorporated. However, it is still challenging to realize InGaAs with wavelength of over $2.0 \mu \mathrm{m}$ through MOCVD because of its high growth temperature. Owing to the low growth temperature combined with the accurate control of growth rate and the solid-source beam flux, as well as the convenience of As and $\mathrm{P}$ beam flux ramping via needle valves, SSMBE is technically preferred. In this paper, the structural property of the SSMBE grown $\operatorname{In}_{0.76} \mathrm{Ga}_{0.32}$ As epilayer on a compositionally undulating step-graded InAs $\mathrm{P}_{1-\mathrm{y}}$ buffer with unequal single layer thickness is investigated in detail by using atomic force microscopy (AFM), reciprocal space mapping (RSM), and cross-sectional transmission electron microscopy (TEM). The excitation power dependence of photoluminescence $(\mathrm{PL})$ peak energy at $10 \mathrm{~K}$ and the temperature-dependent photoluminescence from $10 \mathrm{~K}-300 \mathrm{~K}$ are also examined and discussed.

\section{Experiments}

\subsection{Growth of Extended Wavelength InGaAs Structure}

All samples were performed by a Veeco Gen20A all solid-source MBE (SSMBE) system equipped with arsenic (As) and phosphorus (P) cracker cells. The source flux in beam equivalent pressure (BEP) was measured by beam flux meter (BFM). Prior to material growth, surface pretreatment of semi-insulated InP (100) substrate was taken in preparation module at $350{ }^{\circ} \mathrm{C}$ for $1 \mathrm{~h}$, followed by thermal desorption in growth module at $495^{\circ} \mathrm{C}$ (monitored by pyrometer) within $\mathrm{P}_{2}$ ambient for $5 \mathrm{~min}$. A $100 \mathrm{~nm}$ undoped $\mathrm{InP}$ buffer layer was grown at $490{ }^{\circ} \mathrm{C}$ to get a better surface at first, and then the undoped $\operatorname{In} \mathrm{As}_{\mathrm{y}} \mathrm{P}_{1-\mathrm{y}}$ buffer layers were grown at a constant temperature of $475{ }^{\circ} \mathrm{C}$ with a growth rate of 1.5 ML/s under stabilized $\mathrm{P}_{2} \mathrm{BEP}$ with varied $\mathrm{As}_{2}$ BEP. A compositionally undulating stepgraded (CUSG) buffer structure with unequal layer thickness was applied in $\operatorname{In} \mathrm{As}_{\mathrm{y}} \mathrm{P}_{1-\mathrm{y}}$ buffer. Each step consisted of a $30 \mathrm{~nm}$ bottom modulation layer with high As composition and a $180 \mathrm{~nm}$ upper modulation layer with relatively lower As composition. Then, an "overshoot" InAs ${ }_{0.52} \mathrm{P}_{0.48}$ layer, which has lattice misfit extent of $1.7 \%$ with respect to InP, was intentionally grown to further compensate the residual strain, followed by $\operatorname{In} A s_{0.49} \mathrm{P}_{0.51}$ "virtual substrate" growth. The total thickness of InAs $\mathrm{P}_{1-\mathrm{y}}$ buffers was about $2.09 \mu \mathrm{m}$. After that, the extended wavelength $\operatorname{In}_{0.76} \mathrm{Ga}_{0.24}$ As layer growth at $495^{\circ} \mathrm{C}$ with a growth rate of $1.2 \mathrm{ML} / \mathrm{s}$ was grown (noted as sample A after). For comparison, $\operatorname{In}_{0.76} \mathrm{Ga}_{0.24} \mathrm{As}$ on common compositionally step-graded (CSG) $\mathrm{InAs}_{\mathrm{y}} \mathrm{P}_{1-\mathrm{y}}$ buffer (noted as sample B after) with a total thickness of $2.96 \mu \mathrm{m}$ was also grown. Detailed structure was schematically illustrated in Figure 1. 


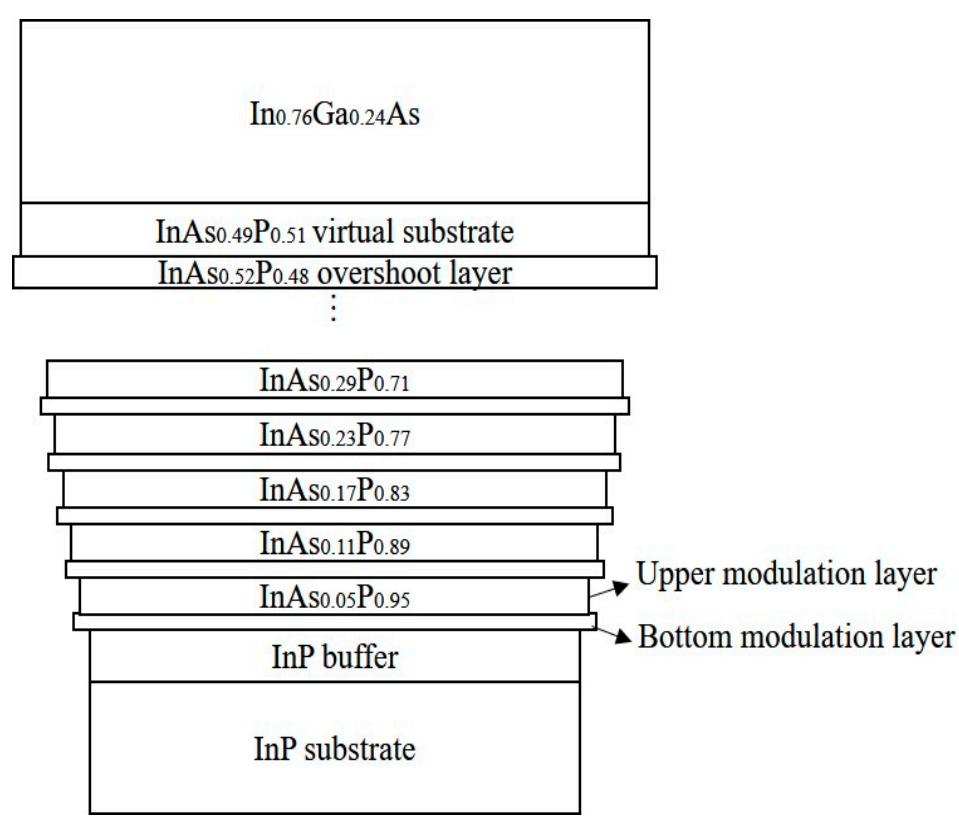

(a)

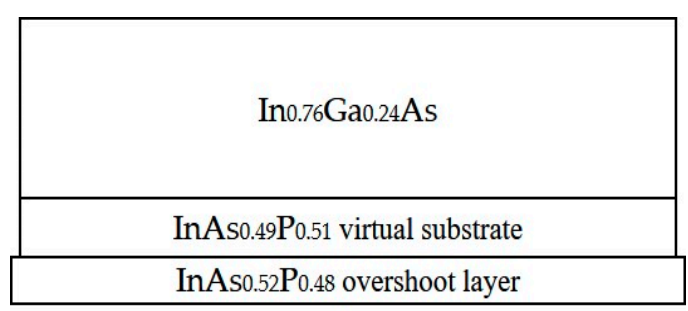

\begin{tabular}{|c|}
\hline InAs0.21P0.79 \\
\hline InAs0.17P0.83 \\
\hline InAs0.13P0.87 \\
\hline InAs0.09P0.91 \\
\hline InAs0.05P0.95 \\
\hline InP buffer \\
\hline InP substrate \\
\hline
\end{tabular}

(b)

Figure 1. (a) Schematic diagram of extended wavelength InGaAs on InP substrate with compositionally undulating step-graded (CUSG) buffer structure (noted as sample A). (b) Schematic diagram of $\operatorname{In}_{0.76} \mathrm{Ga}_{0.24} \mathrm{As}$ on InP substrate with common compositionally step-graded buffer structure (noted as sample B).

\subsection{Measurements of Structural and Optical Properties}

After the completion of epitaxy, surface morphology was characterized by using Bruker Dimension ICON atomic force microscopy (AFM) in tapping mode. The asymmetric strain relaxation properties were checked by reciprocal space mapping (RSMs) using highresolution triple axis XRD via Bruker D8 Discover, then microstructures and defects were evaluated by FEI Talos 20 transmission electron microscope (TEM) operating at $200 \mathrm{kV}$ on cross-sectioned samples, which were prepared by focus ion beam (FIB) microscope (FEI Scios), and PL measurements were conducted on a sample that was mounted on the cold finger of a Janis closed cycle cryostat with a varying temperature range of $10 \mathrm{~K}-300 \mathrm{~K}$. The excitation was $532 \mathrm{~nm}$ laser with chopper, and the emission signal from the sample was dispersed with a Horiba iHR350 monochromator, amplified with lock-in amplifier (SHR380) and detected by InAs detectors.

\section{Results and Discussion}

\subsection{Surface Morphology}

The $20 \mu \mathrm{m} \times 20 \mu \mathrm{m}$ AFM micrographs and related line profiles in [110] direction of two samples are shown in Figure 2. Characteristic undulating morphology with hills and valleys parallel to the intersection of slip planes with the crystal surface, namely, "crosshatch" pattern can be obviously observed. This surface characteristic is typical surface morphology and appeared after plastic strain relaxation in the heteroepitaxy of mismatched zinc-blende structure layers, which means InGaAs is grown in two-dimensional mode with ideal strain relaxation [21]. The root mean square roughness is $1.38 \mathrm{~nm}$ and $3.60 \mathrm{~nm}$ for sample A and sample B, respectively. Furthermore, the peak-to-valley height of sample B is larger than sample A, as shown in Figure 2c. The results indicate that CUSG structure is better-developed and can smoothen the surface more efficiently, similar conclusion was highlighted [8]. It should be stressed that the thickness of the bottom modulation layer in our case is much thinner. Thinner compositional overshoot could remarkably improve structural and optical properties [22]. In this work, every thin bottom modulation $\operatorname{InAs}_{\mathrm{y}} \mathrm{P}_{1-\mathrm{y}}$ layer is compositional overshoot, and lattice is under compressive 
strain, which should result in hills. However, the thickness does not exceed the critical thickness with respect to the former upper modulation layer, so it provides a relatively flat surface for the following upper modulation layer growth. Under the action of alternatively compressive and tensile strain in CUSG structure, superior surface morphology could be achieved. Therefore, one can conclude that the CUSG structure will provide a better surface morphology relating to higher crystalline quality of the $\operatorname{In}_{0.76} \mathrm{Ga}_{0.24}$ As layer, and thus lead to a much lower dislocation density and better optical property in sample A.

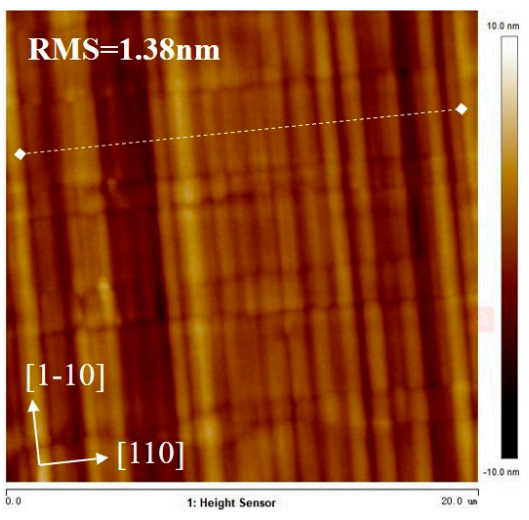

(a)

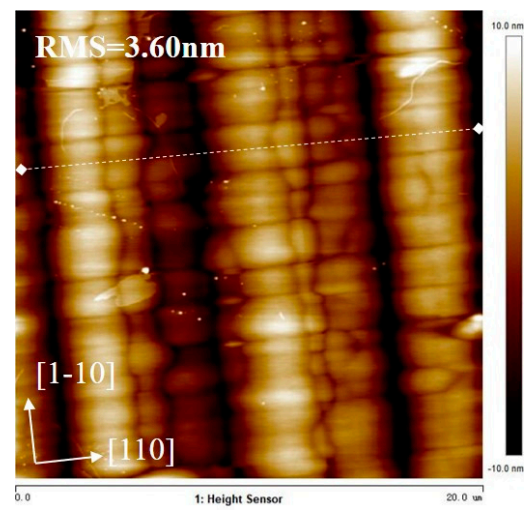

(b)

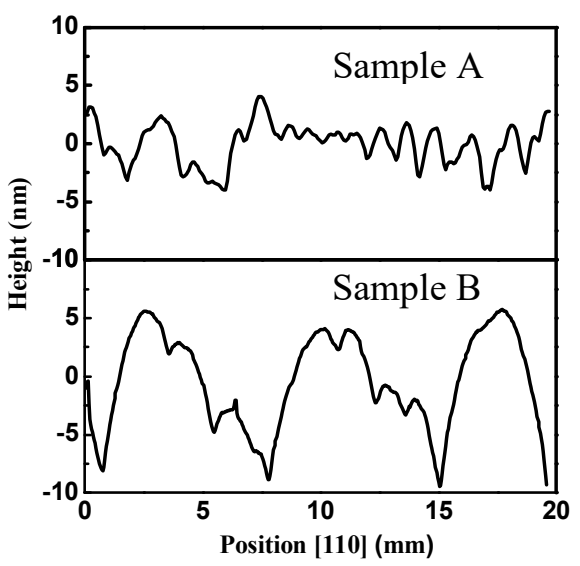

(c)

Figure 2. AFM images of extended wavelength InGaAs on InP substrate at a scanning area of $20 \mu \mathrm{m} \times 20 \mu \mathrm{m}$ : (a) sample A (RMS: $1.38 \mathrm{~nm}$ ); (b) sample B (RMS: $3.60 \mathrm{~nm}$ ); (c) the line profile along [110] direction of sample A and sample B.

\subsection{The Structural Property}

The relaxation state of entire III-V epilayers could be characterized by RSMs. According to the research by Hornstra et al. The equivalent bulk lattice mismatch $f$ can be expressed as $[23,24]$ :

$$
f=\left(f_{\perp}-f_{/ /}\right) \frac{1-v}{1+v}+f_{/ /}
$$

where $f_{\perp}=\frac{\Delta a_{\perp}}{a_{\mathrm{s}}}$, and $f_{/ /}=\frac{\Delta a_{/ /}}{a_{\mathrm{s}}}$ are lattice mismatch in the perpendicular and parallel directions, respectively. The $v$ is the Poisson's ratio, for $\operatorname{In}_{0.76} \mathrm{Ga}_{0.24} \mathrm{As}, v=0.34$ by using elastic constants of $\operatorname{In}_{x} \mathrm{Ga}_{1-x}$ As material at $300 \mathrm{~K}$. The strain relaxation of $\operatorname{In}_{x} \mathrm{Ga}_{1-x} A$ s grown on the InP substrate can be described by the following equation [24]:

$$
R=\frac{a_{/ /}-a_{s}}{a_{\text {relaxed }}-a_{s}}=\frac{f_{/ /}}{f}
$$

where $a_{/ /}$, and $a_{\text {relaxed }}$ represent the in-plane lattice parameters of the epilayer and lattice parameters under completely relaxed state, and $a_{S}$ is the lattice parameters of the substrate.

The RSMs around asymmetric (115) reflection of $\operatorname{In}_{0.76} \mathrm{Ga}_{0.24} \mathrm{As} / \mathrm{InP}$ samples were carried out (as shown in Figure 3). Three distinct reciprocal lattice points (RLP) maxima can be found, which corresponded to (i) the InP substrate; (ii) the uppermost InAsP layer of the InAsy $\mathrm{P}_{1-y}$ buffers; and (iii) the extended wavelength $\operatorname{In}_{0.76} \mathrm{Ga}_{0.24}$ As epilayer, respectively. The vertical solid line represents a fully strained and inclined solid line, which is extended to $Q_{x}=0$ and represents a fully relaxed epilayer. More information about the lattice parameter could be obtained from the position and the intensity distribution of RSMs. By using Equations (1) and (2), the percentage of strain relaxation is $96.47 \%$ and $85.12 \%$ for sample A and sample B, respectively, implying that the $\operatorname{In}_{0.76} \mathrm{Ga}_{0.24}$ As layer and the uppermost InAsP layer almost relaxed completely for sample A. Whereas, residual strain still existed in the uppermost InAsP layer and the $\mathrm{In}_{0.76} \mathrm{Ga}_{0.24}$ As layer of sample B; therefore, some of threading dislocations may penetrate into the InGaAs layer. 


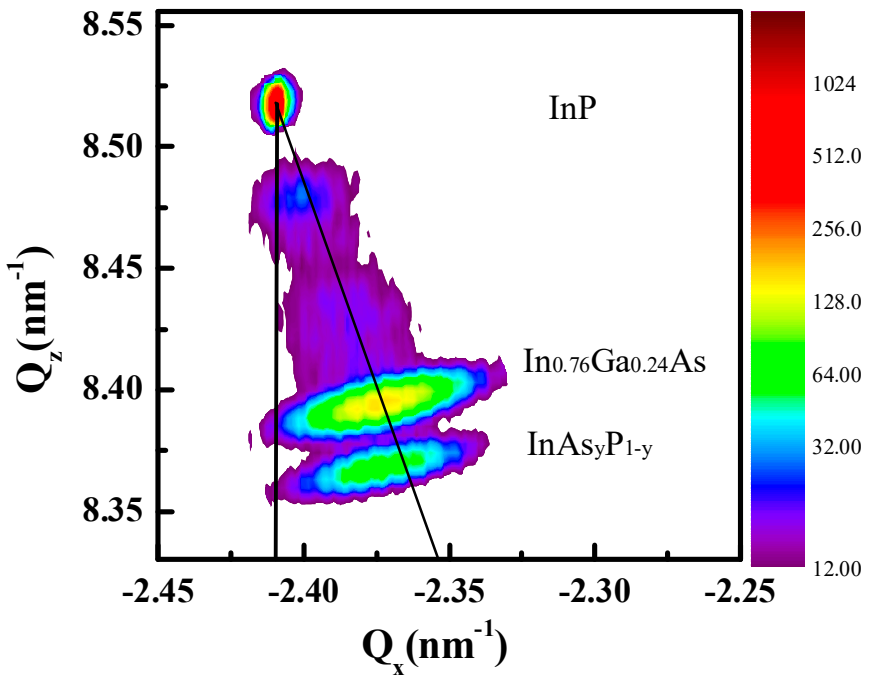

(a)

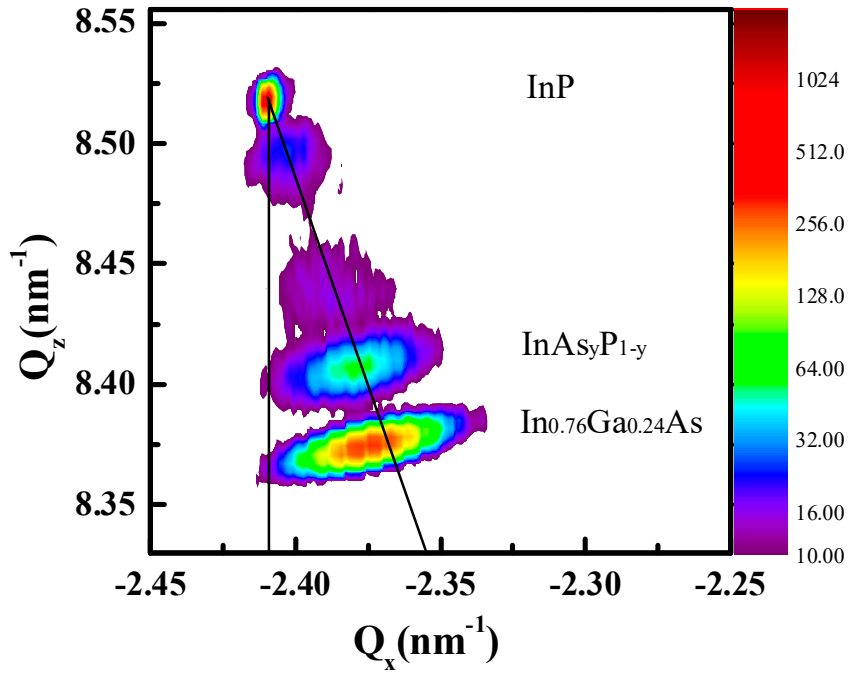

(b)

Figure 3. Asymmetric (115) RSMs of extended wavelength InGaAs on InP substrate: (a) sample A (R = 96.47\%); (b) sample $\mathrm{B}(\mathrm{R}=85.12 \%)$.

Further investigation into the structural property of the two samples is provided by cross-sectional TEM analysis, as shown in Figure 4 , and the $\operatorname{In}_{0.76} \mathrm{Ga}_{0.24}$ As layer and InAs $\mathrm{P}_{1-\mathrm{y}}$ buffer layers are labeled in these figures. Figure $4 \mathrm{a}$ shows the sample grown on the CUSG buffers. Misfit dislocations are restricted along with the internal interface, dislocations are significantly decreased in the uppermost InAsP layer, and no threading dislocation is observed in the $\mathrm{In}_{0.76} \mathrm{Ga}_{0.24}$ As layer of sample A. This mainly benefits from the strain compensation around the interface by the insertion of tensile $\operatorname{InAs}_{\mathrm{y}} \mathrm{P}_{1-\mathrm{y}}$ layers, which can change the dislocation glide direction and facilitate the dislocation annihilation, and the interfaces also prevent the threading dislocations from propagating vertically through the structure [25]. It is a reason of the superior surface and better two-dimensional crosshatch pattern in sample A, as illustrated in AFM analysis. Generally speaking, the TDD decreases with a thicker buffer layer [26], so less defects in sample B should appear regarding a $\sim 0.9 \mu \mathrm{m}$ thicker buffer than that of sample A. However, as shown in Figure $4 \mathrm{~b}$, although most of the dislocations are confined within the $\operatorname{InAs}_{\mathrm{y}} \mathrm{P}_{1-\mathrm{y}}$ buffers for sample $\mathrm{B}$, there are still some dislocations penetrating the bottom of the InGaAs layer. As no other treatment is applied, it is reasonable to conclude that the residual strain contributes to the penetration of dislocations into the InGaAs layer, which is consistent with the RSMs' relaxation analysis discussed above. Moreover, it is also clear that the poor surface morphology of sample B is due to the dislocation penetration result of incomplete relaxation [27].

\subsection{The Optical Property}

The PL spectra of InGaAs epilayer with varying excitation power measured at $10 \mathrm{~K}$ are shown in Figure 5. Single band edge peak is observed in sample A, while an additional low-energy shoulder peak appears in sample B. Such asymmetry may be attributed to localized trap states related to structural defects [12]. For the sake of comparison, PL spectra of sample B are deconvoluted using multiple Gaussian curves, and Figure 5b presents excitation power dependence of band edge peak and shoulder peak. It can be seen from that, the band edge peak keeps almost constant with varying excitation power, although an insignificant red-shift with increasing excitation power is shown in sample A. This may be caused by increasing temperature at the measured point, so sample $A$ is probably sensitive to temperature variation. On the other side, the low-energy shoulder in sample B increases from $0.478 \mathrm{eV}$ to $0.486 \mathrm{eV}$ when the excitation power increases from $33 \mathrm{~mW}$ to $258 \mathrm{~mW}$, and the slight shift of the shoulder peak is also exhibited in In-rich $\operatorname{In}_{x} \mathrm{Ga}_{1-x}$ As samples, which is mainly due to defect-related energy band filling effect [12]. In addition, the intensity 
tends to saturate at high excitation power, which is normally a signature of the localization phenomena for potential fluctuations or carrier transfer in the band-tail [28].

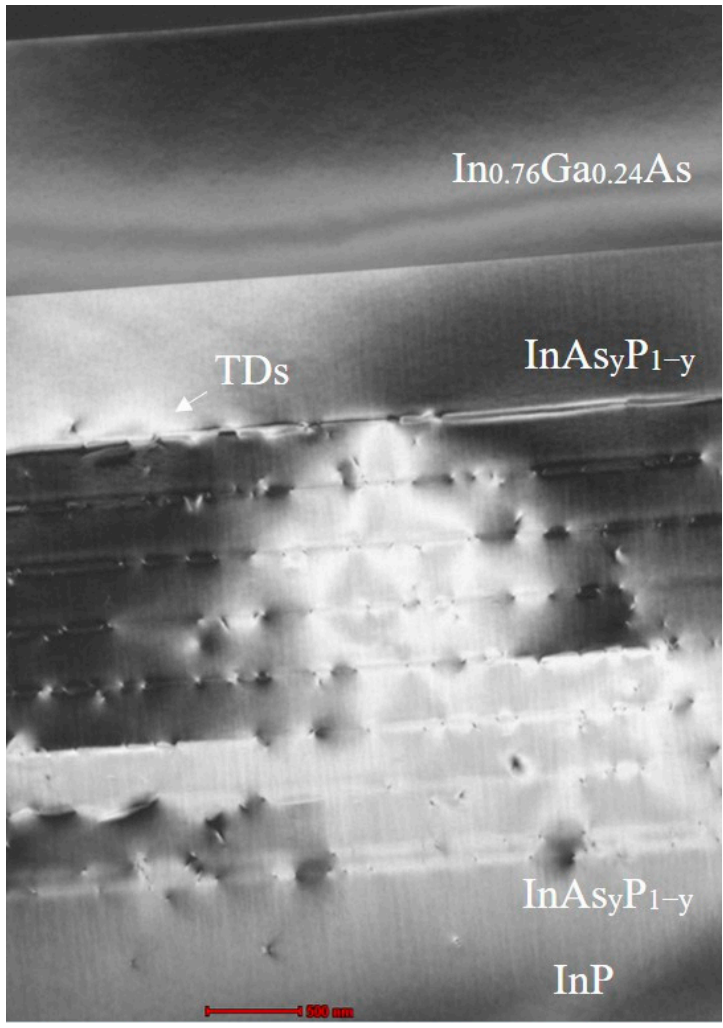

(a)

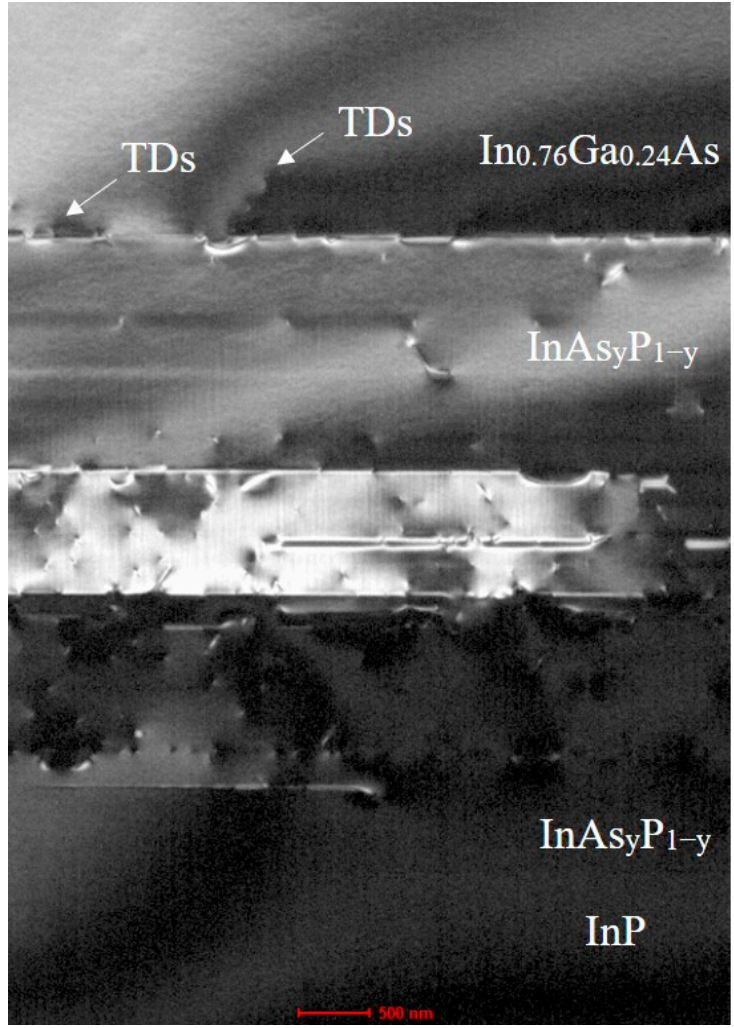

(b)

Figure 4. Cross-sectional TEM images of extended wavelength InGaAs on InP substrate: (a) sample A and (b) sample B.

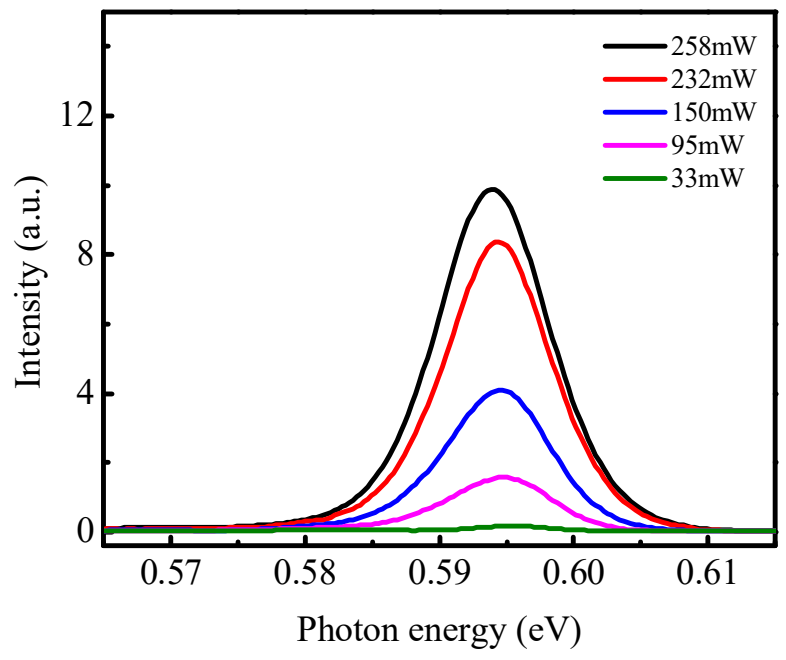

(a)

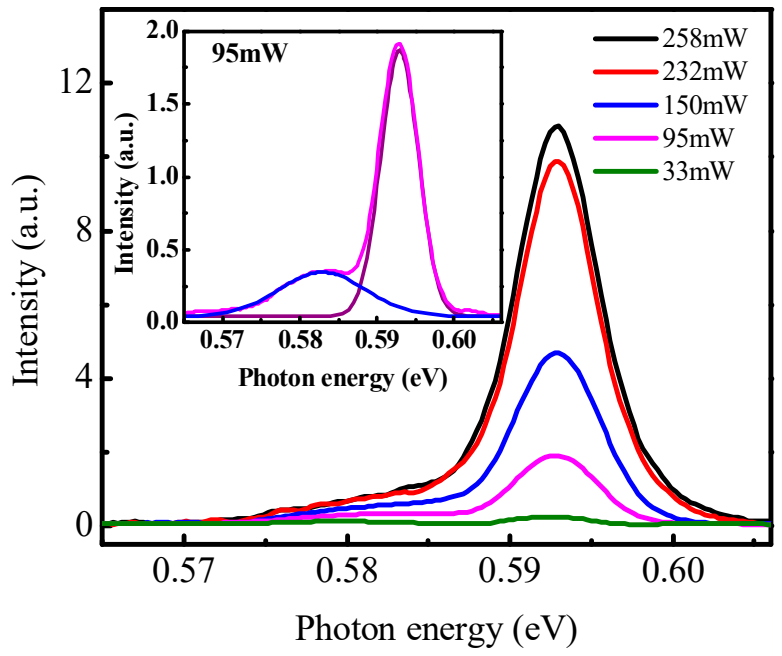

(b)

Figure 5. (a) PL spectra of extended wavelength InGaAs on InP substrate measured at $10 \mathrm{~K}$ (smoothed): (a) sample A and (b) sample B (the inset is Gaussian decomposition of PL spectrum at $95 \mathrm{~mW}$ ).

Figure 6a presents PL spectra measured at room temperature for sample A and sample B, and the peak wavelength is $2297 \mathrm{~nm}$ and $2302 \mathrm{~nm}$, respectively. An asymmetric line shape with a long high-energy tail and relatively faster declining low-energy edge is displayed for both samples, especially for sample A. This kind of profile is usually explained 
by a conventional band-to-band transition model, which predominately reflects the density of states of three-dimensional electronic structure and the Boltzmann distribution of carriers or free excitons [29]. Moreover, it is worth mentioning that the intensity of sample A is about 5 times stronger than that of sample B.

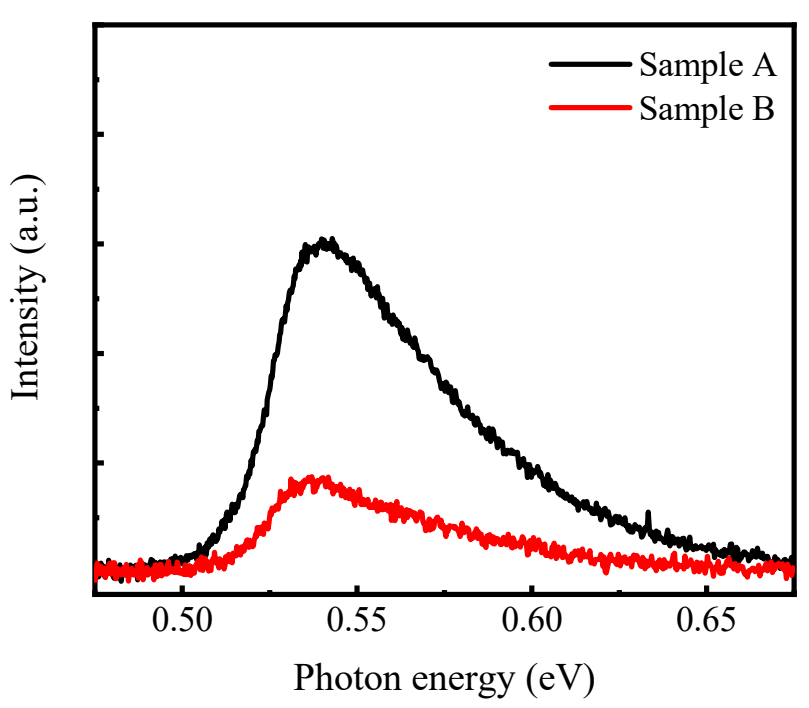

(a)

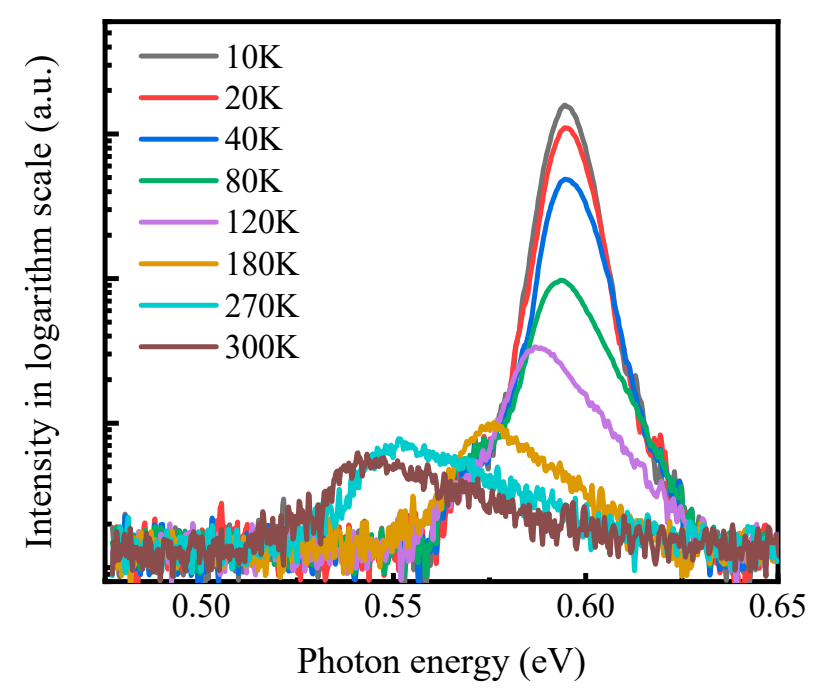

(b)

Figure 6. (a) PL spectra of extended wavelength InGaAs sample at room temperature. (b) PL spectra of extended wavelength InGaAs sample A at different temperatures from $10 \mathrm{~K}$ to $300 \mathrm{~K}$ (Intensity is plotted in logarithm scale).

Temperature-dependent photoluminescence (TDPL) measurements is also performed from $10 \mathrm{~K}$ to $300 \mathrm{~K}$, as shown in Figure $6 \mathrm{~b}$. Peak energy shows red shifted when the temperature is higher than $40 \mathrm{~K}$, which is due to band shrinkage effect (thermal activation of carriers). The peak position and the intensity are extracted from those spectra at each temperature.

The relationship between peak energy and temperature can be described by Varshni's empirical equation as following [30]:

$$
E(T)=E(0)-\frac{\alpha T^{2}}{T+\beta}
$$

where $E(0)$ is the energy gap at $0 \mathrm{~K}, \alpha$ plays the role of $T \rightarrow \infty$ limiting value of band-gap shrinkage coefficient, and $\beta$ is an associated parameter related to the Debye temperature. As mentioned in excitation power-dependent PL, localized states caused by potential fluctuations or carrier transfer in the band-tail may exist. The inhomogeneity of indium composition can result in fluctuation of energy band, thus, an atypical S-shaped behavior of peak energy with temperature will be presented [12]. Unlike the localized states induced Sshaped behavior highlighted in Ref. [12], this work produces good fits to the experimental data for the entire temperature range. The behavior indicates that the distribution of indium composition is homogeneous in the MBE-grown extended wavelength InGaAs material. The fitted equation is also presented in Figure 7, and the Varshni parameter $\alpha$ determined in sample A is higher than that of sample B. A higher $\alpha$ means that sample A is relatively more sensitive with temperature, which is in accordance with the insignificant red-shift with increasing excitation power of sample A [13]. 


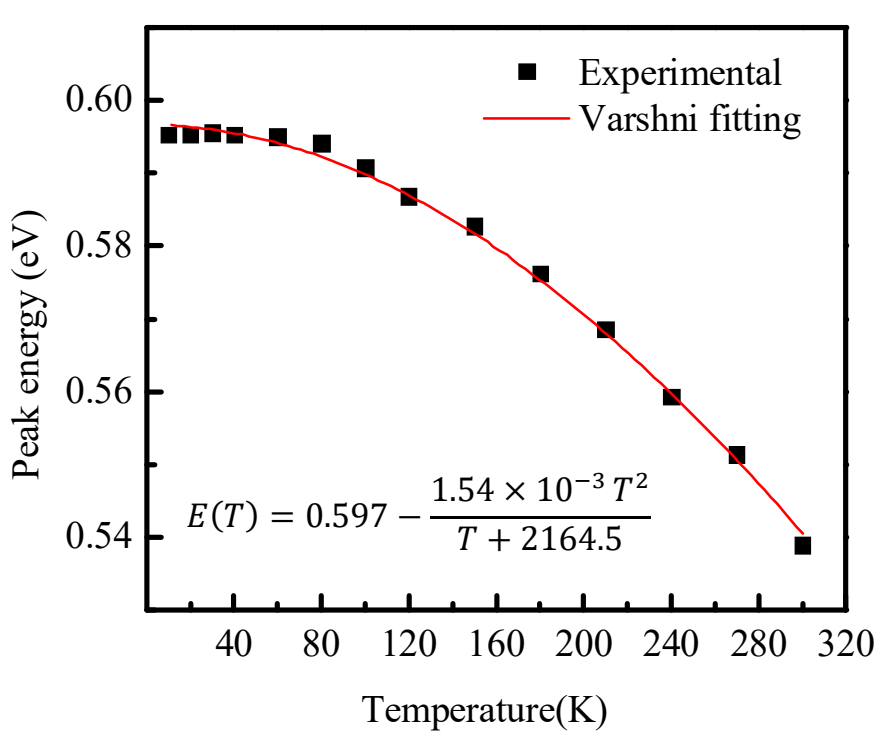

(a)

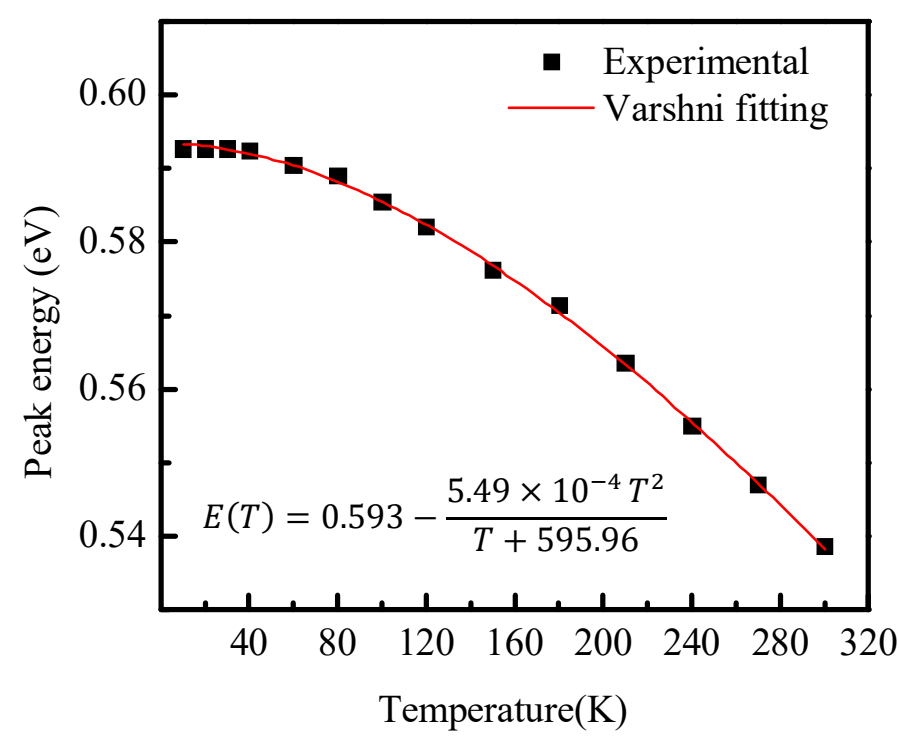

(b)

Figure 7. The peak energy of extended wavelength InGaAs on InP substrate as a function of temperature (a) sample A and (b) sample B, the fitting parameters are also given.

As can also be seen from Figure 6b, the overall intensity of the PL spectra diminishes significantly as temperature increases, which is due to the thermal quenching effect caused by phonon-assisted non-radiative recombination. In order to further understand the mechanism of carrier thermal quenching in the InGaAs layer, Arrhenius-like expression given in Equation (4) is applied to fit the experimental data of the PL intensity [31,32], in which two non-radiative recombination processes are assumed.

$$
I(T)=\frac{I_{0}}{1+C_{1} \exp \left(\frac{-E_{a 1}}{k_{B} T}\right)+C_{2} \exp \left(\frac{-E_{a 2}}{k_{B} T}\right)}
$$

where $T$ is the measured temperature, $I(T)$ is the integrated PL intensity, and $k_{B}$ is Boltzmann's constant. $E_{a 1}$ and $E_{a 2}$ are the thermal activation energy of non-radiative recombination processes. $C_{1}$ and $C_{2}$ are the ratio of non-radiative to radiative probability for the non-radiative mechanism, in other words, they are related to the density of non-radiative recombination centers.

Figure 8 shows the relationship between intensity and the best fit using Equation (4) with the experimental data, and the activation energy obtained from the fit for each sample are also indicated. The value of $E_{a 1}$, which is mainly due to interfacial roughness, is $5.44 \mathrm{meV}$ and $6.24 \mathrm{meV}$ of sample A and sample B, respectively. It indicates that the localized energy caused by interfacial roughness is small. The second process, which is predominant at higher temperatures, mainly results from the suppression of non-radiative recombination centers linked to misfit dislocations, and it is said that higher $E_{a 2}$ values may be associated with improved internal quantum efficiency [13]. In our case, $E_{a 2}$ values are $26.67 \mathrm{meV}$ and $38.03 \mathrm{meV}$ for sample A and sample B, respectively. Sample A seems to perform worse at room temperature. In fact, it is effectively the transition probability $C_{2}$ of the recombination that governs the PL intensity at room temperature [31]. We could see that $C_{2}$ of sample $A$ is about 5 times smaller than that of sample $B$, indicating much less misfit dislocation in sample A, in line with the results evidenced in TEM. Hence, stronger PL intensity at room temperature is displayed. 


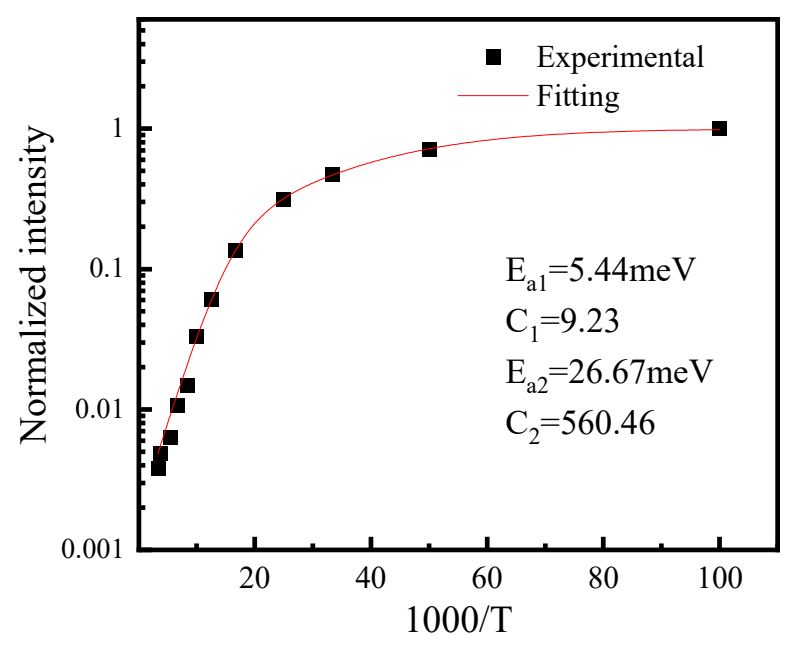

(a)

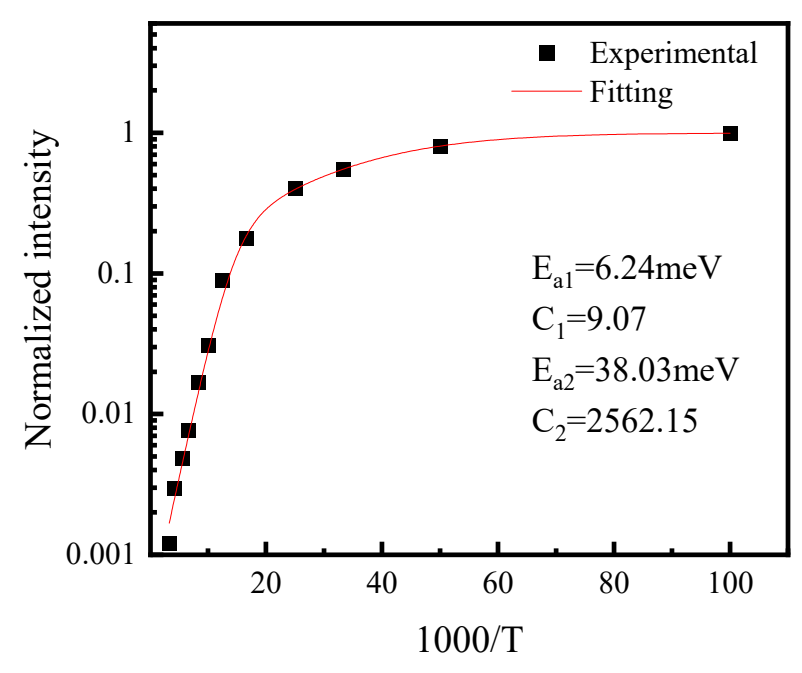

(b)

Figure 8. Variation of the normalized integrated PL intensity with the reciprocal temperature for extended wavelength InGaAs on InP substrate. The red solid lines represent the Arrhenius fitting results: (a) sample A; (b) sample B.

\section{Conclusions}

Extended wavelength $(2.3 \mu \mathrm{m})$ InGaAs on common and compositionally undulating step-graded InAsP buffers have been grown by SSMBE, and their structural and optical properties have been investigated by means of AFM, RSM, TEM, and PL measurements. By taking advantage of the strain compensation mechanism, a smaller surface roughness of $1.38 \mathrm{~nm}$, a better relaxation of $96.47 \%$, and fewer dislocations were presented for the sample with improved CUSG buffer structure and thinner buffers. Furthermore, the roomtemperature PL intensity of InGaAs grown on a CUSG buffer showed $\sim 5$ times stronger than the one grown on a CSG buffer, which could be attributed to less deep non-radiative centers in the $\operatorname{In}_{0.76} \mathrm{Ga}_{0.24}$ As layer. The emission peak energy with varying temperatures was in good agreement with Varshni's empirical equation, which implies high crystal quality without inhomogeneity-induced localized states under a low growth temperature. In addition, the InGaAs grown on a CUSG buffer is slightly more sensitive to temperature. To sum up, our work shows that compositionally undulating step-graded InAsP buffers with a thinner bottom modulation layer, grown by SSMBE, is an effective approach to prepare InGaAs materials with wavelengths longer than $2.0 \mu \mathrm{m}$ and to break the lattice restriction on the materials with even larger mismatch.

Author Contributions: Conceptualization and methodology, X.L. and S.L.; formal analysis and visualization, J.X., T.W., W.Y. and Y.W.; investigation, S.J.; writing original draft preparation, X.L.; writing review and editing, J.X., W.Y. and S.L.; supervision, S.L.; project administration, S.L.; funding acquisition, S.L. All authors have read and agreed to the published version of the manuscript.

Funding: This research was funded by National Key R\&D Program, grant number 2018YFB2003305; National Natural Science Foundation of China, grant numbers $61774165,61875224,61827823,61875224$ and 61827823; SINANO, grant number Y8AAQ11003; and Vacuum Interconnected Nanotech Workstation, grant number B2006.

Data Availability Statement: The data presented in this study are available within the manuscript.

Acknowledgments: The authors thank Platform for Characterization \& Test and Vacuum Interconnected Nanotech Workstation of Suzhou Institute of Nano-Tech and Nano-Bionics (SINANO), Chinese Academy of Sciences (CAS) for the technical support.

Conflicts of Interest: The authors declare no conflict of interest. 


\section{References}

1. Nahory, R.; Pollack, M.; Johnston, W.; Barns, R. Band-gap Versus Composition and Demonstration of Vegards Law for In1xGaxAsyP1-y Lattice Matched to InP. Appl. Phys. Lett. 1978, 33, 659-661. [CrossRef]

2. Kleipool, Q.L.; Jongma, R.T.; Gloudemans, A.M.S.; Schrijver, H.; Lichtenberg, G.F.; van Hees, R.M.; Maurellis, A.N.; Hoogeveen, R.W.M. In-Flight Proton-Induced Radiation Damage to SCIAMACHY's Extended-Wavelength InGaAs near-Infrared Detectors. Infrared Phys. Technol. 2007, 50, 30-37. [CrossRef]

3. Dai, P.; Lu, S.; Uchida, S.; Ji, L.; Wu, Y.; Tan, M.; Bian, L.; Yang, H. Room-Temperature Wafer Bonded InGaP/GaAs//InGaAsP/InGaAs Four-Junction Solar Cell Grown by All-Solid State Molecular Beam Epitaxy. Appl. Phys. Express 2016, 9, 016501. [CrossRef]

4. Gamel, M.M.A.; Lee, H.J.; Rashid, W.E.S.W.A.; Ker, P.J.; Yau, L.K.; Hannan, M.A.; Jamaludin, M.Z. A Review on Thermophotovoltaic Cell and Its Applications in Energy Conversion: Issues and Recommendations. Materials 2021, 14, 4944. [CrossRef] [PubMed]

5. Wu, Y.; Ji, L.; Dai, P.; Tan, M.; Lu, S.; Yang, H. Effects of Buffer Layer and Back-Surface Field on MBE-Grown InGaAsP/InGaAs Solar Cells. Jpn. J. Appl. Phys. 2016, 55, 022301. [CrossRef]

6. Zhang, J.; Itzler, M.A.; Zbinden, H.; Pan, J.-W. Advances in InGaAs/InP Single-Photon Detector Systems for Quantum Communication. Light Sci. Appl. 2015, 4, e286. [CrossRef]

7. Hudait, M.K.; Brenner, M.; Ringel, S.A. Metamorphic $\operatorname{In}_{0.7} \mathrm{Al}_{0.3} \mathrm{As} / \mathrm{In}_{0.69} \mathrm{Ga}_{0.31} \mathrm{As}$ Thermophotovoltaic Devices Grown on Graded InAs $_{y} P_{1-y}$ Buffers by Molecular Beam Epitaxy. Solid-State Electron. 2009, 53, 102-106. [CrossRef]

8. Ji, L.; Lu, S.L.; Zhao, Y.M.; Tan, M.; Dong, J.R.; Yang, H. Compositionally Undulating Step-Graded InAs $\mathrm{P}_{1-y}$ Buffer Layer Growth by Metal-Organic Chemical Vapor Deposition. J. Cryst. Growth 2013, 363, 44-48. [CrossRef]

9. Ji, L.; Lu, S.-L.; Jiang, D.-S.; Zhao, Y.-M.; Tan, M.; Zhu, Y.-Q.; Dong, J.-R. 0.6-EV Bandgap In0.69Ga0.31As Thermophotovoltaic Devices with Compositionally Undulating Step-Graded InAs $\mathrm{P}_{(1-y)}$ Buffers. Chin. Phys. B 2013, 22, 026802. [CrossRef]

10. Hudait, M.; Lin, Y.; Palmisiano, M.; Ringel, S. 0.6-EV Bandgap In0.69Ga0.31As Thermophotovoltaic Devices Grown on InAsyP1-y Step-Graded Buffers by Molecular Beam Epitaxy. IEEE Electron. Device Lett. 2003, 24, 538-540. [CrossRef]

11. Besikci, C. Extended Short Wavelength Infrared FPA Technology: Status and Trends. In Proceedings of the Quantum Sensing and Nano Electronics and Photonics XV, San Francisco, CA, USA, 28 January-2 February 2018; Razeghi, M., Brown, G., Lewis, J., Leo, G., Eds.; SPIE: Bellingham, WA, USA, 2018; Volume 10540.

12. Smiri, B.; Ben Arbia, M.; Ilkay, D.; Saidi, F.; Othmen, Z.; Dkhil, B.; Ismail, A.; Sezai, E.; Hassen, F.; Maaref, H. Optical and Structural Properties of In-Rich $\mathrm{In}_{\mathrm{x}} \mathrm{Ga}_{1-\mathrm{X}}$ As Epitaxial Layers on (100) InP for SWIR Detectors. Mater. Sci. Eng. B-Adv. Funct. Solid-State Mater. 2020, 262, 114769. [CrossRef]

13. Mehdi, H.; Martin, M.; Jany, C.; Virot, L.; Hartmann, J.M.; Da Fonseca, J.; Moeyaert, J.; Gaillard, P.; Coignus, J.; Leroux, C.; et al. Monolithically Integrated InGaAs/AlGaAs Multiple Quantum Well Photodetectors on 300 Mm Si Wafers. AIP Adv. 2021, 11, 085028. [CrossRef]

14. Zhang, Y.-G.; Gu, Y.; Wang, K.; Li, A.-Z.; Li, C. Properties of Gas Source Molecular Beam Epitaxy Grown Wavelength Extended InGaAs Photodetector Structures on a Linear Graded InAlAs Buffer. Semicond. Sci. Technol. 2008, 23, 125029. [CrossRef]

15. Zimmermann, L.; John, J.; Degroote, S.; Borghs, G.; Van Hoof, C.; Nemeth, S. Extended Wavelength InGaAs on GaAs Using InAlAs Buffer for Back-Side-Illuminated Short-Wave Infrared Detectors. Appl. Phys. Lett. 2003, 82, 2838-2840. [CrossRef]

16. Gu, Y.; Zhang, Y.-G.; Li, A.-Z.; Wang, K.; Li, C.; Li, Y.-Y. Structural and Photoluminescence Properties for Highly StrainCompensated InGaAs/InAlAs Superlattice. Chin. Phys. Lett. 2009, 26, 077808.

17. Hidouri, T.; Saidi, F.; Al-Shahri, B.M. Tuning Spontaneous Emission in BInGaAs/GaAs QWs by Varying the Growth Temperature: Above 1.2 Mm Emission and Solar Cells Application. Opt. Quantum Electron. 2021, 53, 532. [CrossRef]

18. Saidi, H.; Zitouni, O.; Ridene, S. Investigation of Orientation Dependence of Piezoelectric Effects in Strained GaAs/InGaAs Quantum Well Laser. Mater. Sci. Eng. B 2021, 273, 115400. [CrossRef]

19. Gu, Y.; Zhang, Y.G.; Wang, K.; Fang, X.; Li, C.; Zhou, L.; Li, A.Z.; Li, H. Effects of Growth Temperature and Buffer Scheme on Characteristics of InP-Based Metamorphic InGaAs Photodetectors. J. Cryst. Growth 2013, 378, 65-68. [CrossRef]

20. Hudait, M.; Lin, Y.; Palmisiano, M.; Tivarus, C.; Pelz, J.; Ringel, S. Comparison of Mixed Anion, InAs $\mathrm{y}_{1-\mathrm{y}}$ and Mixed Cation, $\mathrm{In}_{\mathrm{X}} \mathrm{Al}_{1-\mathrm{X}}$ As Metamorphic Buffers Grown by Molecular Beam Epitaxy on (100)InP Substrates. J. Appl. Phys. 2004, 95, 3952-3960. [CrossRef]

21. Andrews, A.; LeSar, R.; Kerner, M.; Speck, J.; Romanov, A.; Kolesnikova, A.; Bobeth, M.; Pompe, W. Modeling Crosshatch Surface Morphology in Growing Mismatched Layers. Part II: Periodic Boundary Conditions and Dislocation Groups. J. Appl. Phys. 2004, 95, 6032-6047. [CrossRef]

22. Fang, X.; Gu, Y.; Zhang, Y.G.; Zhou, L.; Wang, K.; Li, H.S.B.Y.; Liu, K.H.; Cao, Y.Y. Effects of Compositional Overshoot on InP-Based InAlAs Metamorphic Graded Buffer. J. Infrared Millim. Waves 2013, 32, 481. [CrossRef]

23. Hornstra, J.; Bartels, W. Determination of Lattice-Constant of Epitaxial Layers of III-V Compounds. J. Cryst. Growth 1978, 44, 513-517. [CrossRef]

24. Tseng, M.-C.; Horng, R.-H.; Wuu, D.-S.; Yang, M.-D. Effect of Crystalline Quality on Photovoltaic Performance for $\operatorname{In}_{0.17} \mathrm{Ga}_{0.83} \mathrm{As}$ Solar Cell Using X-Ray Reciprocal Space Mapping. IEEE J. Quantum Electron. 2011, 47, 1434-1442. [CrossRef]

25. He, Y.; Yan, W.; Sun, Y.; Dong, J. Improved Quality of InP Layer on GaAs Substrates by Using Compositionally Modulated Step-Graded AlGaInAs Buffers. J. Mater. Sci. Mater. Electron. 2019, 30, 16251-16256. [CrossRef] 
26. Romanov, A.; Pompe, W.; Beltz, G.; Speck, J. Modeling of Threading Dislocation Density Reduction in Heteroepitaxial Layers. I. Geometry and Crystallography. Phys. Status Solidi B-Basic Res. 1996, 198, 599-613. [CrossRef]

27. Ahrenkiel, S.P.; Wanlass, M.W.; Carapella, J.J.; Ahrenkiel, R.K.; Johnston, S.W.; Gedvilas, L.M. Optimization of Buffer Layers for Lattice-Mismatched Epitaxy of $\mathrm{Ga}_{x} \mathrm{In}_{1-x} \mathrm{As} / \mathrm{InAs}_{\mathrm{y}} \mathrm{P}_{1-\mathrm{y}}$ Double-Heterostructures on InP. Sol. Energy Mater. Sol. Cells 2007, 91, 908-918. [CrossRef]

28. Hidouri, T.; Parisini, A.; Ferrari, C.; Orsi, D.; Baraldi, A.; Vantaggio, S.; Nasr, S.; Bosio, A.; Pavesi, M.; Saidi, F.; et al. Combined Impact of B2H6 Flow and Growth Temperature on Morphological, Structural, Optical, and Electrical Properties of MOCVD-Grown B(In)GaAs Heterostructures Designed for Optoelectronics. Appl. Surf. Sci. 2021, 151884, in press. [CrossRef]

29. Su, Z.C.; Xu, S.J.; Wang, R.X.; Ning, J.Q.; Dong, J.R.; Lu, S.L.; Yang, H. Electroluminescence Probe of Internal Processes of Carriers in GaInP Single Junction Solar Cell. Sol. Energy Mater. Sol. Cells 2017, 168, 201-206. [CrossRef]

30. Varshni, Y. Temperature Dependence of Energy Gap in Semiconductors. Physica 1967, 34, 149-154. [CrossRef]

31. Lambkin, J.; Considine, L.; Walsh, S.; Oconnor, G.; Mcdonagh, C.; Glynn, T. Temperature-dependence of the Photoluminescence Intensity of Ordered and Disordered $\operatorname{In}_{0.48} \mathrm{Ga}_{0.52}$ P. Appl. Phys. Lett. 1994, 65, 73-75. [CrossRef]

32. Zhang, X.; Yang, W.; Xing, Z.; Qiu, H.; Gu, Y.; Bian, L.; Lu, S.; Qin, H.; Cai, Y.; Suzuki, Y.; et al. Investigation of Micromorphology and Carrier Recombination Dynamics for InGaN/GaN Multi-Quantum Dots Grown by Molecular Beam Epitaxy. Crystals 2021, 11, 1312. [CrossRef] 